\title{
RIEMANN-HURWITZ FORMULA FOR MORITA-MUMFORD CLASSES AND SURFACE SYMMETRIES
}

\author{
NariYa KaWAZUmi AND TAKeshi Uemura
}

\begin{abstract}
Let a finite group $G$ act on a compact Riemann surface $C$ in a fathful and orientation preserving way. Then we describe the Morita-Mumford classes $e_{n}\left(C_{G}\right) \in H^{2 n}(G ; Z)$ of the homotopy quotient (or the Borel construction) $C_{G}$ of the action in terms of fixedpoint data. This fixed-point formula is deduced from a higher analogue of the classical Riemann-Hurwitz formula based on computations of Miller [Mi] and Morita [Mo].
\end{abstract}

\section{Introduction}

The last two decades have witnessed a remarkable progress of cohomological study of the mapping class group for a surface. D. Mumford $[\mathrm{Mu}]$ and $\mathrm{S}$. Morita [Mol] independently defined a series of cohomology classes of the mapping class group, whose zeroth term is equal to the Euler number of the surface up to sign. J. Harer has been obtaining various significant results including the homology stability of the mapping class groups [H1]. The Morita-Mumford classes play an important role in the stable cohomology ring of the mapping class groups. It has been revealed by Arbarello, Cornalba, Harer, Miller, Morita and others [AC] [H2-3] [Mi] [Mol-4] [KM]. The torsion part of the cohomology of the mapping class groups has been highly studied by homotopy theorists including Benson, Charney, Cohen, Lee, Tillmann and Xia [BC] [CC] [CL] [T] [X1,2]. It is notable that Glover and Mislin [GM] have proved there exists a nontrivial torsion class in the $4 n$-dimensional stable cohomology group for each $n \geq 1$ by evaluating the even Chern classes of $G L(Z)$ on torsion elements of the mapping class groups.

In the context of surface bundles the Morita-Mumford classes are defined as follows. Let $\pi: X \rightarrow B$ be an oriented fiber bundle whose fiber is a 2-dimensional connected closed oriented smooth manifold. We call such a bundle briefly a surface bundle. The relative tangent bundle $T_{X / B}$ is the oriented real 2-dimensional vector bundle over the total space $X$ consisting of all the tangent vectors along the fibers. The $n$-th Morita-Mumford class $e_{n}$ is, by

1991 Mathematics Subject Classification. Prımary, 57R20. Secondary, 14H15, 20J06, 32G15, 55R40, 57M20, 57S17

Received May 28, 1998; revised June 16, 1998. 
definition, the Gysin image of the $n+1$-th power of its Euler class $e:=$ $e\left(T_{X / B}\right) \in H^{2}(X ; Z)$

$$
e_{n}=e_{n}(X):=\pi_{1}\left(e^{n+1}\right) \in H^{2 n}(B ; \boldsymbol{Z}),
$$

which is equal to the pull-back of $e_{n}$ by the holonomy homomorphism of $\pi_{1}(B)$ into the mapping class group. Here and throughout this paper we denote the Euler class of an oriented real vector bundle $\eta$ by $e(\eta)$. If $n=0, e_{0}$ is equal to the Euler number of the fibers.

The purpose of the present paper is to study Morita-Mumford classes on finite subgroups of the mapping class groups. We give an explicit formula for the Morita-Mumford classes evaluated on an arbitrary finite subgroup of the mapping class groups in terms of fixed-point data (Theorem B). The authors hope their explicit formula would be widely used to study the torsion part of the cohomology of the mapping class groups.

Our fixed-point formula is deduced from a general formula of MoritaMumford classes for fiberwise branched coverings of surface bundles. Miller [Mi] and Morita [Mol], inspired by Atiyah [A], Hirzebruch [Hi] and Kodaira [Ko], computed Morita-Mumford classes of iterated cyclic coverings to prove the stable algebraic independence of $e_{n}$ 's. Following their computations, we prove it under a certain transversality condition (Theorem A) in $\$ 1$.

Let $\pi_{X}: X \rightarrow B$ and $\pi_{Y}: Y \rightarrow B$ be two surface bundles, and $h: X \rightarrow Y$ a continuous map compatible with the projections. Suppose the map $h$ restricted to each fiber is an orientation preserving branched covering. Denote by $R$ the subset of $X$ consisting of all the ramification points of the map $h$. Now we assume a transversality condition that the restriction of $\pi_{X}$ to $R$ is a locally trivial fibration, and that there exists a fiber preserving homeomorphism $t$ : $D\left(\left.T_{X / B}\right|_{R}\right) \hookrightarrow X$ onto an open neighborhood of $R$ such that $t\left(0_{x}\right)=x$ for all $x \in R$. We call it a fiberwise tubular neighborhood of $R$. Here we denote by $D(\eta)$ the open unit disk bundle associated with a vector bundle $\eta$ with respect to a suitable metric. Let $R_{1}, R_{2}, \ldots, R_{m}$ be the connected components of $R$, and $b_{i}$ the ramification degree along $R_{l}$. Then

THEOREM A. In the situation stated above we have

$$
e_{n}(X)=(\operatorname{deg} h) e_{n}(Y)+\sum_{t=1}^{m}\left(1-b_{i}^{n+1}\right)\left(\left.\pi_{X}\right|_{R_{t}}\right)_{1}\left(e\left(\left.T_{X / B}\right|_{R_{t}}\right)^{n}\right) \in H^{2 n}(B ; Z)
$$

for any $n \geq 1$, where $\operatorname{deg} h \in Z$ is the fiberwise mapping degree of $h$, and $\left(\left.\pi_{X}\right|_{R_{l}}\right)_{1}$ : $H^{*}\left(R_{i} ; \boldsymbol{Z}\right) \rightarrow H^{*}(X ; \boldsymbol{Z})$ is the Gysin map associated with the fibration $\left.\pi_{X}\right|_{R_{l}}$

It also holds for the case $n=0$. In fact, since $e_{0}$ is the Euler number of the fibers, the formula

$$
e_{0}(X)=(\operatorname{deg} h) e_{0}(Y)+\sum_{i=1}^{m}\left(1-b_{i}\right) \cdot \sharp\left(\text { the fiber of }\left.\pi_{X}\right|_{R_{t}}\right) .
$$


is exactly the classical Riemann-Hurwitz formula. This is the reason why we regard Theorem $\mathrm{A}$ as a higher analogue of the Riemann-Hurwitz formula.

If the surface bundles $\pi_{X}$ and $\pi_{Y}$ are $C^{\infty}$-surface bundles, $h$ is a $C^{\infty}$ map, and the ramification locus $R$ is a $C^{\infty}$-submanifold of $X$ transverse to the fibers, then the transversality condition stated above is satisfied.

As another typical example satisfying the transversality condition we have Morita-Mumford classes on finite subgroups of the mapping class groups. In view of the affirmative solution of the Nielsen realization problem by Kerckhoff $[\mathrm{Ke}]$ any finite subgroup of the mapping class group is realized as a holomorphic automorphism group of a suitable Riemann surface. Therefore we may consider the following situation.

Let $G$ be a finite group and $C$ a closed oriented connected 2-dimensional smooth manifold. Suppose $G$ acts on $C$ in a faithful and orientation preserving way. Then we may regard $G$ as a subgroup of the mapping class group. The universal principal $G$-bundle $E_{G} \rightarrow B_{G}$ induces the homotopy quotient (or the Borel construction) of the action $\pi: C_{G} \rightarrow B_{G}$. The space $C_{G}$ is, by definition, the quotient of $E_{G} \times C$ by the diagonal action of $G$. The map $\pi$ induced by the first projection provides an oriented fiber bundle with fiber $C$

$$
C \longrightarrow C_{G} \stackrel{\pi}{\longrightarrow} B_{G} .
$$

Its Morita-Mumford class $e_{n}\left(C_{G}\right) \in H^{*}\left(B_{G} ; Z\right)=H^{*}(G ; Z)$ is equal to the restriction of $e_{n}$ to the subgroup $G$.

If $\pi_{X}$ is the surface bundle $\pi: C_{G} \rightarrow B_{G}, \pi_{Y}$ the product bundle $B_{G} \times$ $(C / G) \rightarrow B_{G}$, and $h: C_{G} \rightarrow B_{G} \times(C / G)$ the canonical projection, then the transversality condition is satisfied. Therefore Theorem A implies the following fixed-point formula as is shown in $\$ 2$.

Denote the isotropy group at a point $p \in C$ by $G_{p}$. The exceptional set

$$
S:=\left\{p \in C ; G_{p} \neq\{1\}\right\}
$$

is a $G$-stable finite subset of $C$, since the action is faithful and orientation preserving. Let $\xi_{p}$ be the oriented real 2-dimensional vector bundle over $B_{G_{p}}$ associated with the action of $G_{p}$ on the tangent space $T_{p} C$ and $e\left(\xi_{p}\right) \in H^{2}\left(B_{G_{p}} ; Z\right)$ $=H^{2}\left(G_{p} ; \boldsymbol{Z}\right)$ its Euler class. Since the transfer map $\operatorname{cor}_{G_{p}}^{G}: H^{*}\left(G_{p} ; \boldsymbol{Z}\right) \rightarrow$ $H^{*}(G ; \boldsymbol{Z})$ is invariant under conjugation, the cohomology class $\operatorname{cor}_{G_{p}}^{G}\left(e\left(\xi_{p}\right)^{n}\right) \in$ $H^{2 n}(G ; \boldsymbol{Z})$ is constant on each $G$-orbit.

THEOREM B. In the situation stated above we have

$$
e_{n}\left(C_{G}\right)=\sum_{p \in S / G} \operatorname{cor}_{G_{p}}^{G}\left(e\left(\xi_{p}\right)^{n}\right) \in H^{2 n}\left(B_{G} ; \boldsymbol{Z}\right)=H^{2 n}(G ; \boldsymbol{Z})
$$

for any $n \geq 1$.

The right-hand side depends only on the isotropy groups and their actions on the tangent spaces at the exceptional points. Our formula may be regarded as a 
certain kind of fixed-point formulas of characteristic classes. Especially if the action is free, the Morita-Mumford classes $e_{n}\left(C_{G}\right)$ vanish for all $n \geq 1$.

\section{Riemann-Hurwitz formula for Morita-Mumford classes}

In this section we shall prove Theorem A following Miller [Mi] and Morita [Mo]. Let $\pi_{X}: X \rightarrow B, \pi_{Y}: Y \rightarrow B, h: X \rightarrow Y, R$ and $R_{l}$ be as in Introduction. We abbreviate as $e_{X}:=e\left(T_{X / B}\right), e_{Y}:=e\left(T_{Y / B}\right)$ and $\eta_{i}:=\left.T_{X / B}\right|_{R_{i}}$. From the transversality condition we have a fiberwise tubular neighborhood $t_{l}: D\left(\eta_{i}\right) \rightarrow X$. Since $\left\{X-R, t_{i}\left(D\left(\eta_{l}\right)\right) ; 1 \leq i \leq m\right\}$ is an open covering of $X$, we obtain an excision isomorphism

$$
t^{*}: H^{*}(X, X-R) \stackrel{\cong}{\longrightarrow} \bigoplus_{i=1}^{m} H^{*}\left(E\left(\eta_{i}\right), E_{0}\left(\eta_{i}\right)\right) .
$$

Here $E\left(\eta_{i}\right)$ is the total space of the bundle $\eta_{i}$ and $E_{0}\left(\eta_{i}\right)=E\left(\eta_{i}\right)$ - (zero section).

Let $\phi_{i}: H^{*-2}\left(R_{l}\right) \rightarrow H^{*}\left(E\left(\eta_{i}\right), E_{0}\left(\eta_{i}\right)\right)$ be the Thom isomorphism associated with $\eta_{i}$, and $U_{i} \in H^{2}(X, X-R)$ such as $t^{*}\left(U_{i}\right)=\phi_{i}(1) . H^{2}(X, X-R)$ is $Z$-free with free basis $\left\{U_{1}, U_{2}, \ldots, U_{m}\right\}$. Clearly we have

$$
t^{*}\left(U_{i} U_{J}\right)= \begin{cases}\phi_{i}\left(e\left(\eta_{i}\right)\right), & \text { if } i=j \\ 0, & \text { if } i \neq j .\end{cases}
$$

Since $T_{X / B}$ is isomorphic to $h^{*} T_{Y / B}$ on $X-R$, the difference $e_{X}-h^{*} e_{Y}$ is in the image of the inclusion homomorphims $j^{*}: H^{2}(X, X-R) \rightarrow H^{2}(X)$, i.e., we have $e_{X}-h^{*} e_{Y}=j^{*}\left(\sum_{l=1}^{m} a_{i} U_{i}\right) \in H^{2}(X)$ for some $a_{i} \in Z$. Restricting it to each $R_{l}$, we obtain

$$
e\left(\eta_{i}\right)-b_{i} e\left(\eta_{i}\right)=a_{i} e\left(\eta_{i}\right) \in H^{2}\left(R_{l}\right)
$$

where $b_{i}$ is the ramification degree along $R_{l}$. From (1.1) and (1.2)

$$
\begin{aligned}
e_{X}^{n+1} & =h^{*} e_{Y}^{n+1}+j^{*}\left(\sum_{l=1}^{m} \sum_{k=1}^{n+1}\left(\begin{array}{c}
n+1 \\
k
\end{array}\right)\left(\left.h^{*} e_{Y}\right|_{R_{i}}\right)^{n+1-k} a_{i}^{k} U_{l}^{k}\right) \\
& =h^{*} e_{Y}^{n+1}+j^{*}\left(\sum_{l=1}^{m} \sum_{k=1}^{n+1}\left(\begin{array}{c}
n+1 \\
k
\end{array}\right) b_{i}^{n+1-k} a_{i}^{k} e\left(\eta_{i}\right)^{n} U_{i}\right) \\
& =h^{*} e_{Y}^{n+1}+j^{*}\left(\sum_{l=1}^{m}\left(1-b_{i}^{n+1}\right) e\left(\eta_{i}\right)^{n} U_{i}\right)
\end{aligned}
$$

for any $n \geq 1$. Hence we obtain

$$
e_{X}^{n+1}=h^{*} e_{Y}^{n+1}+j^{*}\left(t^{*}\right)^{-1}\left(\sum_{t=1}^{m}\left(1-b_{i}^{n+1}\right) \phi_{i}\left(e\left(\eta_{i}\right)^{n}\right)\right)
$$

for $n \geq 1$. 
We denote the fibers of the bundles $\pi_{X}: X \rightarrow B,\left.\pi_{X}\right|_{(X-R)}: X-R \rightarrow B$ and $\left.\pi_{X}\right|_{R_{l}}: R_{l} \rightarrow B$ over a point $s_{0} \in B$ by $C, C^{0}$ and $R_{i} \mid s_{0}$ respectively. The Serre spectral sequence of the pair of fiber bundles $(X, X-R)$ induces an isomorphism

$$
\pi_{X_{*}}: H^{*}(X, X-R) \stackrel{\cong}{\longrightarrow} H^{*-2}\left(B ; H^{2}\left(C, C^{0}\right)\right) .
$$

As a $\pi_{1}(B)$-module, $H^{2}\left(C, C^{0}\right)$ decomposes itself into $\bigoplus_{i=1}^{m} H^{0}\left(R_{i} \mid s_{0}\right)$. The cohomology group $H^{*-2}\left(B ; H^{0}\left(R_{i} \mid s_{0}\right)\right)$ is naturally isomorphic to $H^{*-2}\left(R_{l}\right)$, and the integration map $H^{0}\left(R_{i} \mid s_{0}\right) \rightarrow Z$ induces the Gysin map $\left(\left.\pi_{X}\right|_{R_{l}}\right)_{1}: H^{*-2}\left(R_{l}\right) \rightarrow$ $H^{*-2}(B)$. Therefore we obtain a commutative diagram

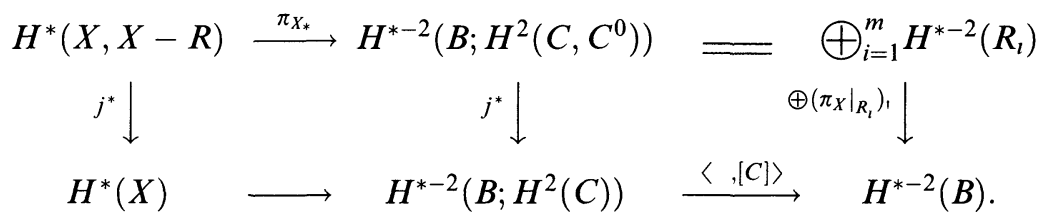

The composite of the lower arrows is equal to the Gysin map $\pi_{X !}$ in the definition of the Morita-Mumford classes, and that of the upper ones equal to $\left(\bigoplus \phi_{i}^{-1}\right) \circ t^{*}$. Consequently, from (1.3), we obtain

$$
\begin{aligned}
e_{n}(X) & =\pi_{X^{\prime}}\left(e_{X}^{n+1}\right)=\pi_{X !}\left(h^{*} e_{Y}^{n+1}+j^{*}\left(t^{*}\right)^{-1}\left(\sum_{t=1}^{m}\left(1-b_{i}^{n+1}\right) \phi_{i}\left(e\left(\eta_{i}\right)^{n}\right)\right)\right) \\
& =(\operatorname{deg} h) e_{n}(Y)+\left(\oplus\left(\left.\pi_{X}\right|_{R_{l}}\right)_{!}\right) \pi_{X_{*}}\left(t^{*}\right)^{-1}\left(\sum_{t=1}^{m}\left(1-b_{i}^{n+1}\right) \phi_{i}\left(e\left(\eta_{i}\right)^{n}\right)\right) \\
& =(\operatorname{deg} h) e_{n}(Y)+\sum_{l=1}^{m}\left(1-b_{i}^{n+1}\right)\left(\left.\pi_{X}\right|_{R_{l}}\right)_{1}\left(e\left(\eta_{i}\right)^{n}\right) .
\end{aligned}
$$

This completes the proof of Theorem A.

\section{Fixed-point formula for surface symmetries}

In order to prove Theorem $\mathrm{B}$, fix a complete system of representatives $\left\{p_{1}, p_{2}, \ldots, p_{m}\right\} \subset S$ with respect to the action of $G$. We abbreviate as $G_{i}=G_{p_{i}}$ and $\xi_{I}=\xi_{p_{i}}$. The quotient $E_{G} / G_{i}$ can serve as the classifying space $B_{G_{i}}$.

Consider the canonical projection

$$
h: C_{G}=\left(E_{G} \times C\right) / G \rightarrow B_{G} \times(C / G), \quad(x, z) \bmod G \mapsto(x \bmod G, z \bmod G) .
$$

The homotopy quotient $S_{G}:=\left(E_{G} \times S\right) / G$ may be regarded as a closed subset of $C_{G}$, and coincides with the ramification locus $R$ of the branched covering $h$. Clearly $\left.\pi\right|_{S_{G}}: S_{G} \rightarrow B_{G}$ is a locally trivial fibration.

Each representative $p_{i}$ corresponds to a connected component of $R$. We introduce a map $f_{l}: B_{G_{l}} \rightarrow C_{G}$ ("multi-valued section of $\pi$ ") by $f_{i}\left(x \bmod G_{i}\right):=$ $\left(x, p_{i}\right) \bmod G$. From a $G$-stable decomposition $S=\coprod_{l=1}^{m} G \cdot p_{i}$ we find $S_{G}=$ 
$\coprod_{l=1}^{m} f_{i}\left(B_{G_{l}}\right)$. Choose a sufficiently small $G_{i}$-stable open disk $D_{i} \subset C$ centered at each $p_{l}$. The map defined by

$$
t_{l}:\left(E_{G} \times D_{i}\right) / G_{i} \rightarrow C_{G}, \quad(x, z) \bmod G_{i} \mapsto(x, z) \bmod G
$$

can be regarded as a tubular neighborhood of the connected component $f_{l}\left(B_{G_{l}}\right)$. Thus the branched covering $h$ satisfies the transversality condition in Theorem $\mathrm{A}$.

The relative tangent bundle $T_{C_{G} / B_{G}}$ is equal to the homotopy quotient of the tangent bundle $T C, T_{C_{G} / B_{G}}=\left(E_{G} \times T C\right) / G$. Hence the disk bundle $\left(E_{G} \times D_{i}\right) / G_{i}$ is isomorphic to the unit disk bundle of $\xi_{l}=\left(E_{G} \times T_{p_{l}} C\right) / G_{i}$. Clearly $e\left(T_{B_{G} \times(C / G) / B_{G}}\right)^{n+1}=0$ for $n \geq 1$. Since $b_{i}=\sharp G_{i}$, we have $b_{i} e\left(\xi_{l}\right)=0 \in$ $H^{2}\left(B_{G_{l}}\right)$. Therefore Theorem A implies

$$
e_{n}\left(C_{G}\right)=\sum_{l=1}^{m}\left(\left.\pi\right|_{f_{l}\left(B_{G_{l}}\right)}\right)_{l}\left(e\left(\xi_{l}\right)^{n}\right)
$$

for $n \geq 1$.

Conceptually a transfer map results from a Gysin map. In this section, however, we replace the Gysin map $\left(\left.\pi\right|_{f_{i}\left(B_{G_{l}}\right)}\right)$, with the transfer map $\operatorname{cor}_{G_{l}}^{G}$ in an explicit manner. For we believe it will be good for future actual computations.

Consider the relative cohomology $H^{2}\left(C, C^{0}\right)$, where $C^{0}=C-S$. Let $v_{i} \in H_{2}\left(C, C^{0}\right)$ be the image of the positive generator of $H_{2}\left(D_{i}, D_{i}-\left\{p_{i}\right\}\right)$. The evaluation at $v_{i}$ induces a $G$-isomorphism

$$
v_{*}: H^{2}\left(C, C^{0}\right) \stackrel{\cong}{\longrightarrow} \bigoplus_{l=1}^{m} \operatorname{Hom}_{\boldsymbol{Z}\left[G_{l}\right]}(\boldsymbol{Z}[G], \boldsymbol{Z})
$$

by the universal mapping property of coinduced modules, and an isomorphism

$$
v_{*}: H^{*-2}\left(B_{G} ; H^{2}\left(C, C^{0}\right)\right) \stackrel{\cong}{\longrightarrow} \bigoplus_{l=1}^{m} H^{*-2}\left(B_{G_{l}} ; \boldsymbol{Z}\right)
$$

by Shapiro's Lemma (cf. e.g., Brown [B].) Therefore we have a commutative diagram

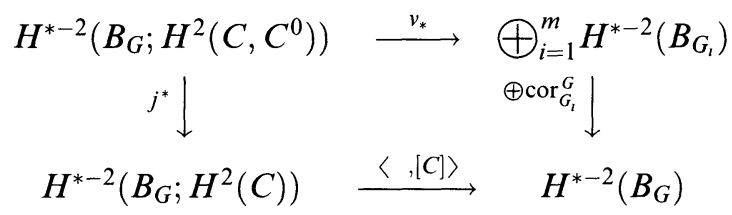

from the definition of the transfer map $\operatorname{cor}_{G_{i}}^{G}$. Comparing it with the commutative diagram (1.4), we obtain

$$
e_{n}\left(C_{G}\right)=\sum_{l=1}^{m}\left(\left.\pi\right|_{f_{l}\left(B_{G_{l}}\right)}\right)_{1}\left(e\left(\xi_{l}\right)^{n}\right)=\sum_{l=1}^{m} \operatorname{cor}_{G_{l}}^{G}\left(e\left(\xi_{l}\right)^{n}\right)
$$

for $n \geq 1$. This completes the proof of Theorem B. 


\section{Applications}

Let $G$ be a perfect finite subgroup of the mapping class group of genus $g$, and $C$ a compact Riemann surface on which $G$ acts as a holomorphic automorphism group. Then, for any element $\gamma \in G$, we have

$$
e_{1}\left(C_{\langle\gamma\rangle}\right)=0 \in H^{2}(\langle\gamma\rangle ; \boldsymbol{Z}),
$$

since $H^{2}(G ; \boldsymbol{Z})=\operatorname{Ext}_{\boldsymbol{Z}}^{1}\left(H_{1}(G ; \boldsymbol{Z}), \boldsymbol{Z}\right)=0$. Here we denote by $\langle\gamma\rangle$ the subgroup of the mapping class group generated by $\gamma$. Therefore we obtain

LEMMA. If $e_{1}\left(C_{\langle\gamma\rangle}\right) \neq 0 \in H^{2}(\langle\gamma\rangle ; Z)$, there is no perfect finite subgroup containing $\gamma$ in the mapping class group.

As an example, consider two complex plane curves

$$
w^{2}=1-z^{2 g+1}, \quad w_{1}^{2}=z_{1}\left(z_{1}^{2 g+1}-1\right)
$$

for $g \geq 1$. Glueing them each other by the map $z_{1}=z^{-1}, w_{1}=z^{-g-1} w$, we obtain a hyperelliptic curve $C$ of genus $g \cdot \zeta:=\exp (2 \pi \sqrt{-1} /(2 g+1))$ defines an automorphism of the curve by $z \mapsto \zeta z$ and $w \mapsto w$. It induces an element $\gamma$ of order $2 g+1$ of the mapping class group of genus $g$. Let $u_{0} \in H^{2}(\langle\gamma\rangle ; \boldsymbol{Z})$ be the Euler class associated with the complex 1-dimensional $G$-module given by multiplication by $\zeta$. $u_{0}^{n}$ generates the group $H^{2 n}(\langle\gamma\rangle ; \boldsymbol{Z}) \cong \boldsymbol{Z} /(2 g+1)$ for each $n$. Then Theorem B implies

$$
e_{n}\left(C_{\langle\gamma\rangle}\right)=u_{0}^{n}+u_{0}^{n}+\left((-g-1) u_{0}\right)^{n}=\left(2+g^{n}\right) u_{0}^{n} \in H^{2 n}(\langle\gamma\rangle ; \boldsymbol{Z})
$$

for any $n \geq 1$. Especially $e_{1}\left(C_{\langle\gamma\rangle}\right) \neq 0$ if $g \geq 2$. Hence the element $\gamma$ is not contained in any perfect finite subgroup of the mapping class group of genus $g \geq 2$ from the lemma stated above. Moreover we obtain $e_{1}^{n} \neq 0$ for any $n \geq 1$ as a torsion element of the cohomology group of the mapping class group of genus $g$, provided that $2 g+1$ is not a power of 3 .

The second author has found some finite cyclic subgroups satisfying $e_{\text {odd }}=0$ and $e_{2} \neq 0$. Moreover he has found a cyclic subgroup satisfying $e_{1}=e_{2}=0$ and $e_{3} \neq 0$. The details will be appear elsewhere. It would be interesting that there would exist a finite subgroup satisfying $e_{1}=e_{2}=\cdots=e_{n-1}=0$ and $e_{n} \neq 0$ for each $n \geq 4$.

Acknowledgements. The authors would like to express their sincere gratitude to Professor S. Morita for valuable comments and encouragement, and to Doctors Y. Kasahara and T. Morifuji for helpful discussions.

\section{REFERENCES}

[AC] E. Arbarello AND M. Cornalba, Calculatıng cohomology groups of moduli spaces of curves via algebraic geometry, preprint. 
[A] M. F ATIYAH, The signature of fibre-bundles, Global Analysis (Papers in honor of K. Kodarra), University of Tokyo Press, Tokyo, 1969, 73-84.

[BC] D. J. Benson AND F R. Cohen, Mapping class groups of low genus and their cohomology, Mem. Amer. Math. Soc., 443 (1991).

[B] K. S. Brown, Cohomology of Groups, Springer, New York-Heidelberg-Berlin, 1982.

[CC] R. M. Charney AND F R. COHEN, A stable splitting for the mapping class group, Michıgan Math. J., 35 (1988), 269-284.

[CL] R. M. ChaRney AND R. LeE, An application of homotopy theory to mapping class groups, J. Pure Appl. Algebra, 44 (1987), 127-135.

[GM] H. Glover and G. Mislin, Torsion in the mapping class group and its cohomology, J. Pure Appl. Algebra, 44 (1987), 177-189.

[H1] J. HARER, Stability of the homology of the mapping class group of orientable surfaces, Ann. of Math., 121 (1985), 215-249.

[H2] J. HARER, The second homology group of the mapping class group of an orientable surface, Invent. Math., 72 (1983), 221-239.

[H3] J. Harer, The third homology group of the moduli of curves, Duke Math. J., 65 (1991), $25-55$.

[Hi] F Hirzebruch, The signature of ramified coverıngs, Global Analysis (Papers in honor of K. Kodarra), University of Tokyo Press, Tokyo, 1969, 253-265.

[I] T. IZAwa, Chern number formula for ramified coverings, Master Thesis, Hokkando Unıversity, 1997 A summary is in: Sūrikaısekikenkyūsho Kōkyūroku, 1033 (1998), 7 20.

[Ka] N. Kawazumi, Homology of hyperelliptic mapping class groups for surfaces, Topology Appl., 76 (1997), 203-216.

[KM] N. Kawazumi AND S. Morita, The primary approximation to the cohomology of the moduli space of curves and cocycles for the stable cohomology classes, Math. Res. Lett., 3 (1996), 629-641.

[Ke] S. P Kerckhoff, The Nielsen realization problem, Ann. of Math., 117 (1983), 235265 .

[Ko] K. KodalRA, A certain type of urregular algebraic surface, J. Anal. Math., 19 (1967), 207215.

[Mi] E. Y MiLler, The homology of the mapping class group, J. Differential Geom., 24 (1986), $1-14$.

[Mf] T. MorifuJI, The $\eta$-invariant of mapping tori with finite monodromies, Topology Appl., 75 (1997), 41-49.

[Mo1] S. Morita, Characteristic classes of surface bundles, Invent. Math., 90 (1987), 551-577

[Mo2] S. Morita, Families of Jacobian manifolds and characteristic classes of surface bundles, I, Ann. Inst. Fourier (Grenoble), 39 (1989), 777-810.

[Mo3] S. MoRITA, Families of Jacobian manifolds and characteristic classes of surface bundles, II, Math. Proc. Cambridge Philos. Soc., 105 (1989), 79-101.

[Mo4] S. MoRita, The extension of Johnson's homomorphism from the Torelli group to the mapping class group, Invent. Math., 111 (1993), 197-224.

[Mu] D. Mumford, Towards an enumerative geometry of the moduli space of curves, Arthmetic and Geometry. Prog. Math., 36, Birkhäuser, Boston, 1983, 271-328.

[T] U. TillmanN, A splittıng for the stable mapping class group, Math. Proc. Cambridge Philos. Soc., to appear.

[X1] Y XIA, The $p$-torsion of the Farrell-Tate cohomology of the mapping class group $\Gamma_{(p-1) / 2}$, Topology '90, Ohıo State Unıv. Math. Res. Inst. Publ., 1, Walter de Gruyter, Berlin, 1992, 391-398. 
[X2] Y XIA, The $p$-torsion of the Farrell-Tate cohomology of the mapping class group $\Gamma_{p-1}, \quad J$. Pure Appl. Algebra, 78 (1992), 319-334.

DEPARTMENT of Mathematics

FACULTY OF SCIENCE

HOKKAIDO UNIVERSITY

SAPPORO, 060-0810

JAPAN

e-mail: kawazumı@math.scı.hokuda1.ac.jp

e-mail: t-uemura@math.scr.hokuda1.ac.jp 